\title{
p53, Cyclin D1, p21 (WAF1) and Ki-67 (MIB1) Expression at Invasive Tumour Fronts of Oral Squamous Cell Carcinomas and Development of Local Recurrence
}

\author{
F Sawair ${ }^{1 *}$, Y Hassona ${ }^{1}$, C Irwin ${ }^{2}$, M Stephenson ${ }^{3}$, P Hamilton ${ }^{3}$, P Maxwell ${ }^{3}$, D \\ Gordon $^{3}$, A Leonard ${ }^{3}$, S Napier $^{3}$
}

\begin{abstract}
Background: Expression of p53, cyclin D1, p21 (WAF1) and Ki-67 (MIB1) was evaluated in oral squamous cell carcinoma (OSCC) to test whether levels of these markers at invasive tumour fronts (ITFs) could predict the development of local recurrence. Materials and Methods: Archived paraffin-embedded specimens from 51 patients with T1/T2 tumours were stained immunohistochemically and analysed quantitatively. Local recurrence-free survival was tested with Kaplan-Meier survival plots (log-rank test) using median values to define low and high expression groups and with a Cox's proportional hazards model in which the expression scores were entered as continuous variables. Results: The assessment of expression of all markers was highly reliable, univariate analysis showing that patients with clear surgical margins, with low cyclin D1 and high p21 expression at the ITF had the best local recurrence-free survival. Multivariate analysis showed that these three parameters were independent prognostic factors but that neither 53 nor MIB1 expression were of prognostic value. Conclusions: Assessment of p53, cyclin D1, p21 (WAF1), and Ki-67 (MIB1) at the ITF could help to predict local recurrence in early stage oral squamous cell carcinoma cases.
\end{abstract}

Keywords: Immunohistochemistry - oral squamous cell carcinoma - p53 - cyclin D1 - p21 (WAF1) - Ki-67 (MIB1)

Asian Pac J Cancer Prev, 17 (3), 1243-1249

\section{Introduction}

Despite the advances in treatment over the past 2030 years, there has been no significant improvement in the prognosis for OSCC, with a high incidence of local recurrence as the main cause of this poor outcome (Pisani et al., 1993; Malone et al., 2004; Ferlay et al., 2008; Ferlay et al., 2012) . TNM staging and histopathological grading are considered the main prognostic systems in OSCC, yet patients with similar stages of disease treated in a uniform manner experience a wide range of outcomes, presumably because of differences in the underlying biology of the tumour. This differential in treatment success is emphasised in patients with localised relatively small T1 and T2 tumours, many of whom develop recurrences or late metastasis. There is a pressing need to identify markers of prognosis to facilitate better assessment of tumour behaviour and aid the tailoring of specific treatments for individual patients particularly in patients with $\mathrm{T} 1$ and $\mathrm{T} 2$ lesions.

The development and progression of OSCC are characterised by imbalances of cell proliferation, differentiation and death, all of which are believed to be the result of alterations in cell cycle control. Studies have shown that alterations in cell cycle regulators are found in many kinds of cancer (Rivera et al., 2014). The p53 tumour suppressor gene has a major protective role to play in normal cells by monitoring the integrity of their genome. The product of wild-type p53 prevents uncontrolled cellular proliferation after DNA damage via G1 arrest of the cell cycle. This arrest functions either to provide extra time for DNA repair by mechanisms activated simultaneously by p53 or, if repair fails, to trigger cell suicide by apoptosis (Patel et al., 2013; Marcel et al. 2015). The p 21 protein, which is encoded by the WAF1/ Cip1 gene, is the main downstream effector of wild-type p53 and transcriptionally activates p21 in response to DNA damage and in p53-dependent apoptosis (King et al., 2014; Yan et al., 2015). Cyclin D1 is encoded by CCND1/ PRAD-1 gene on chromosome locus 11q13. Cyclin D1, a putative protooncogene, is believed to accelerate the G1 phase of the cell cycle by binding to cyclin dependent kinase (cdk) 4 or 6 . Therefore, overexpression of Cyclin D1, usually as a result of gene amplification, may force 


\section{F Sawair et al}

the cells into mitosis before DNA repair or replication is completed and to proliferate in the absence of positive growth factors, resulting in both genomic instability and cancer development (Mahdey et al., 2012; Malumbres, 2014). The number of proliferating or cycling cells in the tumour, an important element in tumour growth, can be estimated using the monoclonal antibody Ki-67 (MIB1). This antibody is directed against a nuclear antigen that is present in all phases of the cell cycle except G0 phase (Kilickap et al., 2014; Li et al., 2015).

Previous studies on the prognostic significance of p53, MIB1, p21 and Cyclin D1 in OSCC as determined by immunohistochemistry describe contradictory findings, probably as a result of variations patient selection, source of tissue, specimen processing, precise immunohistochemical techniques and antibodies used, evaluation of the staining, interpretation of data and statistical analysis (Schliephake et al., 2003; Heah et al., 2011; Kilickap et al., 2014; Petric et al., 2014; Shin and Kim, 2014).

The cells of the invasive front of squamous cell carcinoma often display different molecular and morphological characteristics than more superficial parts of the same tumour. In addition to having the highest cell proliferation, recent evidence suggests that the invasive tumour front (ITF) gives the most prognostic information because, it is claimed, it contains the most aggressive subpopulation of tumour cells that ultimately will invade, spread locally and metastasise (Bryne, 1998; Khunamornpong et al., 2013).

The aim of this study was to investigate whether the immunohistochemical expression of the molecular markers p53, Cyclin D1, p21 and MIB1 at the ITF is associated with local recurrence-free survival (LRFS) in patients with T1/T2 OSCC.

\section{Materials and Methods}

\section{Patients}

51 OSCC cases treated at School of Dentistry, Royal Victoria Hospital, Belfast City Hospital, Altnagelvin Area Hospital and Ulster Hospital Dundonald, Northern Ireland, were selected using the following inclusion criteria: (1) patients with T1/T2 tumours, (2) treated primarily by surgery with or without postoperative radiotherapy, (3) a minimum follow-up of thirty months for patients who did not develop local recurrence and were alive at the end of the study and (4) tissue blocks were available for immunohistochemical evaluation. The tumours were graded by two observers (FS and $\mathrm{SN}$ ) using the invasive front grading (IFG) system and staged according to the UICC criteria Bryne, 1998; Hermanek and Sobin, 1987). The status of the surgical margins was classified as positive if invasive carcinoma was present at the margin and close if the tumour was within $2 \mathrm{~mm}$ of the margin and as clear if the tumour further than $2 \mathrm{~mm}$ of the margin. Out of the 51 patients, 25 developed local recurrence within an average of 15.9 months after primary treatment (median 11, range 3-51), 22 patients (88\%) suffered local recurrence within 24 months after treatment. The study has been approved by the Institution Research an Ethics Committee.

\section{Immunohistochemistry}

The following primary antibodies were used: p53 (DO7; Novocastra; Concentration of stock solution (CSS): 35ug/ml, Dilution 1:50); Cyclin D1 (DCS-6; DAKO; CSS: 307ug/ml, Dilution 1:100); p21 (WAF1, 4D10, Novocastra; CSS: 100ug/ml, Dilution 1:20); and Ki-67 (MIB1, Immunotech; CSS: 200ug/ml, Dilution 1:50). For each patient, a formalin-fixed, paraffin-embedded tissue block showing the ITF was selected for immunostaining. Four-micron sections were deparaffinized, rehydrated and treated with $3 \% \mathrm{H} 2 \mathrm{O} 2$ for 10 min to quench endogenous peroxidase activity. The sections were then immersed in $500 \mathrm{ml}$ of $0.01 \mathrm{M}$ sodium citrate buffer ( $\mathrm{pH} 6.0$ ), heated on full power for 22 minutes in a microwave oven $(850 \mathrm{~W})$ to retrieve antigens. Immunohistochemical staining was done using the Envision system (Horseradish Peroxidasebased two-step immunostaining method, DAKO Corp., Carpinteria, USA) (Sabattini et al., 1998). The sections were placed on racks in a humid container and washed with TRIS-buffered saline (TBS) and incubated at room temperature in the primary antibody solutions for 30 minutes. All test antibodies were controlled with type- and species-specific immunoglobulin solutions at equivalent concentrations as negative controls. In each staining batch, OSCCs known to express high levels of the antigens investigated were included as positive controls. After incubation, the slides were washed in TBS, drained and wiped dry and incubated for 30 minutes at room temperature in species-specific anti-mouse peroxidaselabelled polymer of the Envision system. Following incubation, the slides were washed in TBS, drained and wiped dry and Diaminobenzidine (DAB) (DAKO Corp., Carpinteria, USA) was then used to visualise the target antigens. Sections were then washed in TBS, lightly counterstained with Mayer's haematoxylin, dehydrated, cleaned and mounted.

\section{Evaluation of staining}

The slides were assessed in random order by using a set of random numbers. For each antibody, tumour cells were counted in 10 fields at the ITF, restricted to the most peripheral high power fields, using a Kontron Vidas Image Analysis system (Sabattini et al., 1998). The fields were selected by randomly moving the microscope stage along the ITF, while out of focus. All the tumour cells in each field were counted; tumour cells were considered positive for each of the antibodies if there was brown intranuclear DAB staining irrespective of its intensity, while cells without brown staining were considered negative. The labelling index for each field (field LI), was calculated as the total number of positive cells divided by the total number of cells in that field and for each case, the mean value of the 10 readings of the LI (mean LI) was calculated from the 10 field LI values. For statistical analysis, the patients were divided into two groups using the median value of the mean LI as a cut-point; low expression ( $\leq$ the median) and high expression groups ( $>$ the median). To determine the reliability of assessment, 10 out of the 51 cases were selected randomly and the original slides were re-evaluated to determine the field LI and mean LI approximately 4 weeks after the first set of measurements. 


\section{Statistical analysis}

SPSS version 9.0 for Windows was used for statistical analysis. Reliability of counting was tested using both the "repeatability test" and the Spearman's rho correlation coefficient. The repeatability test proposes that the difference between two repeated measurements exceeds a value of 2.83 times the standard deviation in only $5 \%$ of cases $(=2 \sqrt{2}$ (standard deviation 2$)$. The level of significance for repeat measurement correlation was set at $1 \%(\mathrm{p}<0.01)$. The significance of the correlation between the field LI and mean LI was analysed by the Spearman's rho test while the association between the field LI and mean LI and other variables was tested using the Spearman's rho for continuous variables (e.g., age) or by the $\chi^{2}$ test for categorical or nominal variables. LRFS was established as the period of time between primary treatment and the date of local relapse. The Kaplan-Meier method was used to estimate LRFS and the statistical significance was determined by the logrank test. Multivariate analysis was done using forward

Table 1. Patient Characteristics and Summary of Univariate Analysis of LRFS

\begin{tabular}{|c|c|c|c|c|}
\hline \multirow[t]{2}{*}{ Factor } & \multirow[t]{2}{*}{ No. patients (\%) } & \multicolumn{3}{|c|}{ log-rank test } \\
\hline & & $\chi^{2}$ & $\mathrm{df}$ & $\mathrm{P}$ value \\
\hline Age (y) & $63.14 \pm 12.15(39-84) \mathrm{a}$ & 0.8 & 2 & 0.963 \\
\hline$<60$ & $21(41.2)$ & & & \\
\hline $61-70$ & $13(25.5)$ & & & \\
\hline$>70$ & $17(33.3)$ & & & \\
\hline Gender & & 0.02 & 1 & 0.884 \\
\hline Male & $30(58.8)$ & & & \\
\hline Female & $21(41.2)$ & & & \\
\hline Tobacco smoking & & 0.01 & 1 & 0.925 \\
\hline No & $11(21.6)$ & & & \\
\hline Yes & $33(64.7)$ & & & \\
\hline Unknown & $7(13.7)$ & & & \\
\hline Alcohol drinking & & 0.75 & 1 & 0.386 \\
\hline No & $9(17.6)$ & & & \\
\hline Yes & $34(66.7)$ & & & \\
\hline Unknown & $8(15.7)$ & & & \\
\hline CI score b & & 0.74 & 1 & 0.39 \\
\hline 0 & $26(51)$ & & & \\
\hline 4-Jan & $25(49)$ & & & \\
\hline Treatment & & 0.11 & 1 & 0.745 \\
\hline Surgery & $27(52.9)$ & & & \\
\hline Surgery \& radiotherapy & $24(47.1)$ & & & \\
\hline Anatomical site & & 2.31 & 4 & 0.678 \\
\hline Tongue & $19(37.3)$ & & & \\
\hline Floor of mouth & $12(23.5)$ & & & \\
\hline Lower alveolus & $8(15.7)$ & & & \\
\hline $\mathrm{RP} / \mathrm{AP} / \mathrm{SPc}$ & $4(7.8)$ & & & \\
\hline $\mathrm{BM}+\mathrm{Ualv}+\mathrm{HPd}$ & $8(15.7)$ & & & \\
\hline Margin status & & 6.32 & 2 & 0.0423 \\
\hline Clear & $28(54.9)$ & & & \\
\hline Close & $10(19.6)$ & & & \\
\hline Positive & $13(25.5)$ & & & \\
\hline T stage & & 0.7 & 1 & 0.403 \\
\hline $\mathrm{T} 1$ & $28(54.9)$ & & & \\
\hline $\mathrm{T} 2$ & $23(45.1)$ & & & \\
\hline N stage & & 3.23 & 1 & 0.0723 \\
\hline No & 35 (68.6) & & & \\
\hline $\mathrm{N} 1+\mathrm{N} 2$ & $16(31.4)$ & & & \\
\hline TNM stage & & 4.34 & 2 & 0.114 \\
\hline I & $24(47.1)$ & & & \\
\hline II & $11(21.6)$ & & & \\
\hline III+IV & $16(31.4)$ & & & \\
\hline Depth (mm) & & 1.51 & 1 & 0.219 \\
\hline$\leq 10$ & $32(62.7)$ & & & \\
\hline$>10$ & $19(37.3)$ & & & \\
\hline IFG scoree & $9.22 \pm 2.34(5-14) a$ & 0.75 & 1 & 0.387 \\
\hline$<9$ & $22(43.1)$ & & & \\
\hline 39 & $29(56.9)$ & & & \\
\hline
\end{tabular}

a Mean $\pm \mathrm{SD}$ (range), b patient's general condition classified using the Charlson Comorbidity Index $(\mathrm{CI})$ [38], $\mathrm{c} \mathrm{RP} / \mathrm{AP} / \mathrm{SF}=$ retromolar pad, anterior pillar of fauces and soft palate, $\mathrm{d} \mathrm{BM}+\mathrm{Ualv}+\mathrm{HP}=$ buccal mucosa + upper alveolus + hard palate, e Mean score of two assessors. 


\section{F Sawair et al}

stepwise Cox's proportional hazards test using the LIs as continuous variables. The level of significance was set at $5 \%$ level $(\mathrm{p}<0.05)$.

\section{Results}

Patient and tumour characteristics and their prognostic value in terms of LRFS are shown in Table 1.

\section{Reliability of assessment}

The 10 cases passed the "repeatability test" for the expression of p53, MIB1, Cyclin D1 and p21 and showed a highly significant correlation between the first and second measurements for these re-evaluated cases: p53 (rho $=0.980, \mathrm{p}<0.001)$, MIB1 (0.940, $<<0.001)$, Cyclin D1 $(\mathrm{rho}=0.970, \mathrm{p}<0.001)$ and $\mathrm{p} 21(0.920, \mathrm{p}<0.001)$.

\section{p53 expression at the ITF}

p53-positive tumour cells were mainly seen accumulating at the periphery of the invading tumour strands and islands whereas the inner more mature and differentiated cells were negative. A striking accumulation of p53-positive cells was seen in some cases at the ITF compared with more superficial areas. However, heterogeneity in intensity and location of p53-positive cells was noted within and between different tumour specimens. The median value of $\mathrm{p} 53$ expression was $36.8 \%$ (mean 38.2, range 0-94.9). High p53 expression tended to correlate with high MIB1 expression (rho=0.363, $\mathrm{p}=0.009$ ) while no significant correlation between $\mathrm{p} 53$ and either Cyclin D1 or p21 was observed. There was no significant correlation between p53 expression and any of the patient or tumour characteristics listed in Table 1.

\section{MIB1 expression at the ITF}

MIB1-positive cells were noted at the periphery of tumour islands and strands with some tumours exhibiting a predominant MIB1-positivity at the ITF. Other tumours

Table 2. Summary of Univariate Analysis of LRFS for p53, Cyclin D1, p21 and MIB1

\begin{tabular}{|c|c|c|c|c|}
\hline \multirow[t]{2}{*}{ Factor } & & \multicolumn{3}{|c|}{ log-rank test } \\
\hline & & $\chi^{2}$ & $\mathrm{df}$ & P value \\
\hline $\mathrm{p} 53^{\&}$ & $(>36.8 \%$ vs. $\leq 36.8 \%)$ & 0.35 & 1 & 0.553 \\
\hline Cyclin D1\& & $(>14.6 \%$ vs. $\leq 14.6 \%)$ & 4.51 & 1 & 0.0337 \\
\hline $\mathrm{p} 21^{\&}$ & $(>16.5 \%$ vs. $\leq 16.5 \%)$ & 7.66 & 1 & 0.0056 \\
\hline MIB1\& & $(>25.7 \%$ vs. $\leq 25.7 \%)$ & 2.15 & 1 & 0.143 \\
\hline
\end{tabular}

\&Divided into two categories depending on the value of the median (high $>$ median and low $\leq$ median)

Table 3. Multivariate Cox Proportional Hazard Test for LRFS

\begin{tabular}{lcrccc}
\hline Variables & Beta (b) & Wald & df & p-value & Exp (b) \\
\hline Cyclin D1 $^{\&}$ & 0.0269 & 7.641 & 1 & 0.0057 & 1.027 \\
p21 (WAF1) & -0.0793 & 15.869 & 1 & 0.0001 & 0.924 \\
Margin status* & & 9.526 & 2 & 0.0085 & \\
Close & 1.116 & 4.273 & 1 & 0.039 & 3.052 \\
Positive & 1.53 & 8.944 & 1 & 0.0028 & 4.616 \\
\hline
\end{tabular}

${ }^{\&}$ Entered as continuous variables; *Clear as reference category showed dispersed focal MIB1-positivity. The median value of MIB1 expression was $25.7 \%$ (mean 29.8, range 1.1-82.1). In addition to the correlation with p53, MIB1 showed a significant positive correlation with Cyclin D1 (rho=0.391, $\mathrm{p}=0.005$ ). Significantly higher MIB1 expression was found in $\mathrm{T} 2$ tumours than in $\mathrm{T} 1(\mathrm{p}=0.04)$, in tumours $>10 \mathrm{~mm}$ in depth than in tumours $\leq 10 \mathrm{~mm}$ in depth $(\mathrm{p}=0.01)$ and in patients with lymph node metastasis at presentation than in those without metastasis $(\mathrm{p}=0.047)$.

\section{Cyclin D1 expression at the ITF}

Cyclin D1 produced a similar staining pattern to that of p53 and MIB1 although occasionally it produced cytoplasmic staining in contrast to the nuclear staining in the case of p53 and MIB1. Similar to p53 and MIB1 some tumours showed predominant Cyclin D1-positive cells at the ITF while some showed focal positively or no discernible pattern. The median value of Cyclin D1 expression was $14.6 \%$ (mean 19.5, range 0.17-76.2). Other than the correlation with $\mathrm{p} 53$, we did not detect any correlation of Cyclin D1 with clinical or pathological parameters.

\section{p21 expression at the ITF}

The intensity of p21 staining was generally weaker than that of p53, MIB1 and Cyclin D1. In contrast to p53, MIB1 and Cyclin D1,p21 labelled the more differentiated central cells adjacent to the keratin pearls leaving the outer proliferating and less well differentiated cells negative. Mitotic figures were unreactive to $\mathrm{p} 21$. The median value

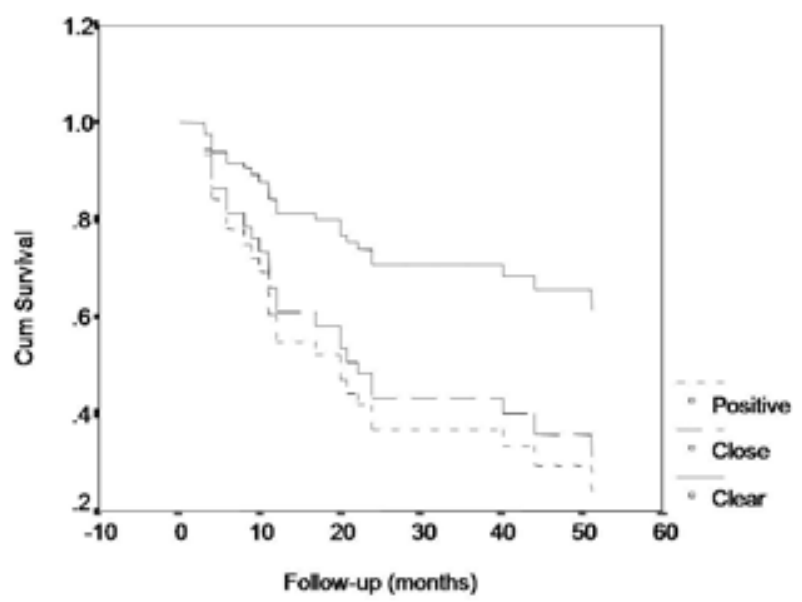

Figure 1. Cumulative LRFS plot for the patients with clear, close and positive surgical margins
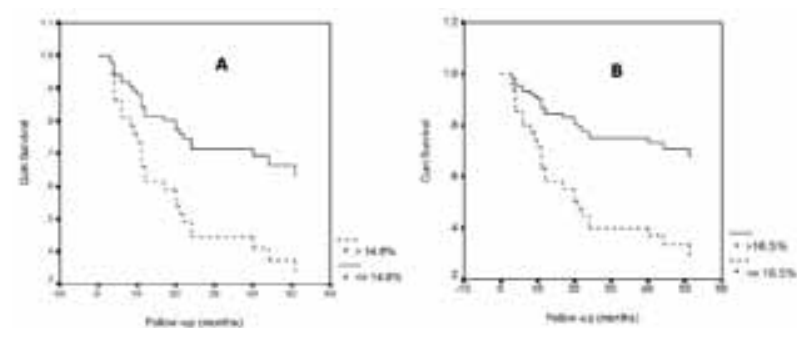

Figure 2. A) Cumulative LRFS for Cyclin D1 Protein Expression. B) Cumulative LRFS for p21 Protein Expression 


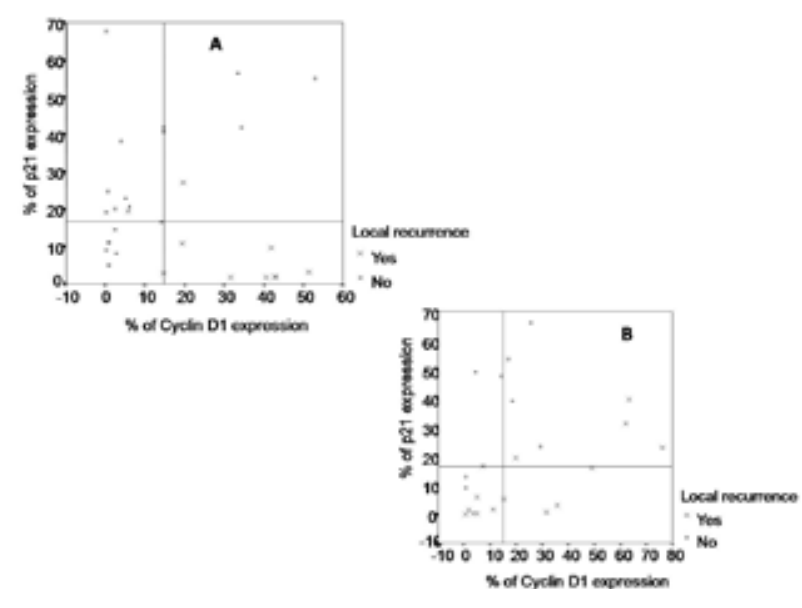

Figure 3. A. The Development or not of Local Recurrence in Patients with Clear Surgical Margins in Relation to the level of Expression of Both p21 and

Cyclin D1. Horizontal \& vertical reference lines = median value of expression of p21 (16.5\%) and Cyclin D1 (14.6\%), respectively. B. The development or not of local recurrence in patients with close/positive surgical margins in relation to the level of expression of both p21 and Cyclin D1. Horizontal $\&$ vertical reference lines $=$ median value of expression of $\mathrm{p} 21$ $(16.5 \%)$ and Cyclin D1 (14.6\%), respectively

of p21 expression was $16.5 \%$ (mean 20.8, range 0.1067.6). No significant correlation was found between $\mathrm{p} 21$ expression and clinical or pathological parameters or with the other markers.

\section{Prognostic factors for local recurrence-free survival}

Univariate analyses (Table $1 \& 2$ ) showed that patients with clear surgical margins ( $\mathrm{p}=0.042$, Figure 1$)$ and those with low Cyclin D1 ( $\mathrm{p}=0.034$, Figure 2A) or high p21 $(p=0.0056$, Figure $2 B$ ) expression at the ITF had the best LRFS. Ten out of twenty-eight patients with clear margins (35.7\%) developed local recurrence compared with 6/10 $(60 \%)$ and $9 / 13(69.2 \%)$ of those with close and positive margins, respectively. Interestingly, in patients who had clear margins, a majority of the those who had high Cyclin D1 developed local recurrence especially those with low p21 while the vast majority of patients who had low Cyclin D1 did not develop local recurrence whether p21 expression was high or low (Figure 3A). In contrast, in patients with close or positive margins the vast majority of patients who had low p21 developed local recurrence whether Cyclin D1 expression was high or low while there was approximately $50 \%$ chance of developing local recurrence when p21 level was high (Figure 3B). Multivariate analysis confirmed that Cyclin D1 and p21 immunohistochemical expression and the status of the surgical margin were significant independent prognostic factors in the prediction of local recurrence (Table 3). Cox's proportional hazards test calculated a hazard function for Cyclin D1 protein expression of 1.027 , indicating an increase in the risk of local recurrence of almost $3 \%$ with every $1 \%$ increase in the protein expression at the ITF while that for p21 was 0.92 , indicating an $8 \%$ decrease in the risk of local recurrence with every $1 \%$ increase in the protein expression. The model also showed that the risk of local recurrence is approximately 3 times greater in patients with close margins and approximately 4.5 times greater in patients with positive margins when compared with patients with clear margins.

\section{Discussion}

Local recurrence is one of the principle reasons for treatment failure in OSCC (Malone et al., 2004). In this study, the selection criteria were chosen to investigate the importance of clinicopathological and some molecular markers in predicting the likelihood of development of local recurrence in T1/T2 tumours; 25 patients who suffered local recurrence were compared with 26 patients who did not develop local relapse. Our results showed that none of the clinicopathological variables studied was associated with local recurrence except the status of the surgical margin. The presence of either a close or a positive surgical margin increased the risk of developing local recurrence markedly, results in keeping with previous reports (Dissanayaka et al., 2012). However, our results showed that around $36 \%$ of patients with clear margins developed local recurrence while $31-40 \%$ of patients with close or positive margins did not, highlighting the need to determine alternative molecular prognostic markers that would help to identify those at high risk of developing recurrence who would benefit from more aggressive therapy or close follow-up. To our knowledge, no published study on patients with OSCC assesses the association between p53, Cyclin D1, MIB1 and p21 protein expression at the ITF and the development of local recurrence. A few previous investigators evaluated the prognostic significance of p53 and MIB1 at the ITF of OSCC but they did not study local recurrence in particular or include measures of reliability in their methodology (Kato et al., 2011; Hassan et al., 2011; Heah et al., 2011; Silva et al., 2013; Verma et al., 2014).

There is no agreement on the threshold level of positive staining that constitutes protein overexpression so that most authors have chosen cut-off points that give prognostically significant statistical differences between patient groups. Altman et al. (1994) stressed the fact that the "optimal cut-off approach", dividing the patients into groups by evaluating the effect of taking several cut-off points and selecting the cut-off that produced the most significant finding in relation to patient's outcome, increases significantly the false-positive rate (i.e., multiple testing bias) (Altman et al., 1994). Therefore, in the univariate analysis in this study the median value was chosen as a cut-off point because the median is less prone to bias being a pre-specified cut-off point. In addition we assessed the expression of the marker as continuous variables in multivariate analysis to determine whether the effect of the differential expression is gradual or linear.

We found high p21 expression at the ITF to be significantly associated with better prognosis in terms of local recurrences and appeared independent of any other clinicopathological variables. The importance of p21 as a prognostic factor was highlighted in the multivariate analysis suggesting that $\mathrm{p} 21$ expression may be related to the biological behaviour of OSCC.p21 is able to interact 
with and inhibit all cdks, thus arresting cells in the G1or G2-phase of the cell cycle (Rani \& Minden, 2014). A high level of p21 expression is necessary to inhibit cell cycle progression as $\mathrm{p} 21$ presents a threshold that cyclincdk complexes must overcome before the cell can enter into S-phase. This may suggest that tumours with low expression of $\mathrm{p} 21$ have lost control of cellular proliferation and may explain why these patients experienced a poor prognosis. However, this function of p21 alone is insufficient to explain the prognostic value of p21 in the current study since no significant correlation was found between the expression of p21 and cell proliferation as detected by MIB1. p21 is involved in p53-dependent induction of apoptosis and can retard the growth of human SCC cell lines in vivo and it may be that low levels of p21 incapacitate the apoptosis pathway (Rani \& Minden, 2014).

We found no correlation between expression of p53 protein at the ITF and any of the clinicopathological parameters or LRFS. Previous reports on p53 expression at the ITF of OSCCs found no significant association with overall survival, although they did not examine the association with the development of local recurrence (Kato et al., 2011; Hassan et al., 2011; Heah et al., 2011; Silva Junior Jde et al., 2013; Verma et al., 2014). Why is it, if p21 is the main downstream effector of $\mathrm{p} 53$, that the expression of p21 was found to be important in prognosis but not p53? There is a number of possible explanations. First, it is not possible using immunohistochemistry alone to determine whether the positive immunoreactivity for $\mathrm{p} 53$ reflects the presence of stable mutant $\mathrm{p} 53$ protein or stabilisation of normal p53 through its binding to certain cellular gene products such as cdc 2 protein kinase, mdm 2 and heat shock proteins or with viral proteins notably E6 of highrisk human papillomaviruses (Mosmann et al., 2015). On the other hand, there will be false-negative staining for p53 when the less common nonsense and frame-shift mutations result in the absence of p53 in the tumour cells. Therefore, p53 immunoexpression does not exactly correspond with p53 gene status. Second, p53 is only one of the regulators of p21; p21 can be induced by other events, such as serum starvation, contact inhibition and certain growth factors without being accompanied by an increase in $\mathrm{p} 53$ protein. The failure to identify a correlation between p 21 and p53 expression immunohistochemically support observations by other authors in favour of the independent action of these proteins (Harada \& Ogden, 2000; Kato et al., 2000; Taghavi et al., 2010; Shinohara et al., 2014).

Our results suggest that tumours with high Cyclin D1 expression at the ITF are more likely to recur locally, independent of the other clinicopathological parameters, including margin status. Previous studies on OSCC, although they did not focus specifically on the ITF, have reported that Cyclin D1 overexpression is associated with reduced disease-free or overall survival (Schliephake et al., 2004). The G1- to S-phase transition in the cell cycle is believed to be accelerated by the binding of Cyclin D1 to cdk4/6, so that Cyclin D1 overproduction, mostly by gene amplification, results in loss of cell cycle control with unchecked cell division and proliferation (Mahdey et al., 2011).
In spite of a significant correlation between MIB1 expression at the ITF and p53 expression, tumour depth, $\mathrm{T}$ - and $\mathrm{N}$-stage of the tumour, we found no correlation with LRFS. The growth of a tumour depends on the proportion of cycling cells, the length of the cell cycle and the rate of cell loss or differentiation; MIB1 only estimates the first of these factors and it is not possible to measure the tumour growth precisely using MIB1-staining alone.

In conclusion, we have shown that the expression of Cyclin D1 and p21 could represent a molecular basis for the biological significance of the ITF in determining the biological behaviour of T1/T2 OSCC as both were independently associated with local recurrence in our patients. These findings suggest that immunohistochemical evaluation of Cyclin D1 and p21 expression may be a further useful prognostic marker for the selection of subgroups of patients at higher risk of developing local recurrence who could benefit from more aggressive surgical approaches, adjuvant radiotherapy or chemotherapy as well as for identifying those who require regular and closer follow-up. However, further studies involving larger number of patients are required.

The status of the surgical margin was also important, obtaining a clear surgical margin is an important determinant of good outcome; however as our results showed some patients with clear margins developed local recurrence while some patients with close/positive margins did not. This may suggest that the assessment of the status of the surgical margins using histopathology alone is a crude method. In this study, we did not assess epithelial dysplasia at the margins due to the reported subjectivity in its diagnosis and the difficulty in achieving reproducible data (Manchanda \& Shetty, 2011). However, we believe that molecular markers could play a more objective and hence more reliable method for the assessment of dysplasia at the margins. Further studies to examine the association between p53, MIB1, Cyclin D1 and p21 expression at the margin of OSCC and the development of local recurrence are required.

\section{References}

Altman DG, Lausen B, Sauerbrei W, et al (1994). Dangers of using "optimal" cutpoints in the evaluation of prognostic factors. J Natl Cancer Inst, 86, 829-35.

Bryne M (1998). Is the invasive front of an oral carcinoma the most important area for prognostication? Oral Dis, 4, 70-7.

Dissanayaka WL, Pitiyage G, Kumarasiri PV, et al (2012). Clinical and histopathologic parameters in survival of oral squamous cell carcinoma. Oral Surg Oral Med Oral Pathol Oral Radiol, 113, 518-25.

Ferlay J, Shin HR, Bray F, et al (2010). Estimates of worldwide burden of cancer in 2008, GLOBOCAN 2008. Int J Cancer, 127, 2893-917.

Ferlay J, Soerjomataram I, Ervik M, Dikshit R, et al (2013). GLOBOCAN 2012 v1.0, cancer incidence and mortality worldwide, IARC cancer base No. 11 [Internet]. Lyon, France, International Agency for Research on Cancer.

Pisani P, Parkin DM, Ferlay J (1993). Estimates of the worldwide mortality from eighteen major cancers in 1985. Implications for prevention and projections of future burden. Int J Cancer, $\mathbf{5 5}, 891-903$.

Harada K, Ogden GR (2000). An overview of the cell cycle arrest 
protein, p21 (WAF1). Oral Oncol, 36, 3-7.

Hassan NM, Hamada J, Kameyama T, et al (2011). Increased expression of the PRL-3 gene in human oral squamous cell carcinoma and dysplasia tissues. Asian Pac J Cancer Prev, 12, 947-51.

Heah KG, Hassan MI, Huat SC (2011). p53 Expression as a marker of microinvasion in oral squamous cell carcinoma. Asian Pac J Cancer Prev, 12, 1017-22.

Hermanek P, Sobin LH (1987) International Union Against Cancer (UICC). TNM classification of malignant tumours. Berlin, Springer-Verlag.

Kato H, Yoshikawa M, Fukai Y, et al (2000). An immunohistochemical study of p16, pRb, p21 and p53 proteins in human esophageal cancers. Anticancer Res, 20, 345-9.

Kato K, Kawashiri S, Yoshizawa K, et al (2011). Expression form of p53 and PCNA at the invasive front in oral squamous cell carcinoma, correlation with clinicopathological features and prognosis. J Oral Pathol Med, 40, 693-8.

Khunamornpong S, Settakorn J, Sukpan K, et al (2013). Prognostic value of pathological characteristics of invasive margins in early-stage squamous cell carcinomas of the uterine cervix. Asian Pac J Cancer Prev, 14, 5165-9.

Kilickap S, Kaya Y, Yucel B, et al (2014). Higher Ki67 expression is associates with unfavorable prognostic factors and shorter survival in breast cancer. Asian Pac J Cancer Prev, 15, 1381-5.

King H, Nicholas NS, Wells CM (2014). Role of p-21-activated kinases in cancer progression. Int Rev Cell Mol Biol, 309, 347-87.

Li LT, Jiang G, Chen Q, et al (2015). Ki67 is a promising molecular target in the diagnosis of cancer. Mol Med Rep, 11, 1566-72.

Mahdey HM, Ramanathan A, Ismail SM, et al (2011). Cyclin D1 amplification in tongue and cheek squamous cell carcinoma. Asian Pac J Cancer Prev, 12, 2199-204.

Malone JP, Stephens JA, Grecula JC, et al (2004). Disease control, survival, and functional outcome after multimodal treatment for advanced-stage tongue base cancer. Head Neck, 26, 561-72.

Malumbres M (2014). Cyclin-dependent kinases. Genome Biol, 15,122 .

Manchanda A, Shetty DC (2012). Reproducibility of grading systems in oral epithelial dysplasia. Med Oral Patol Oral Cir Bucal, 17, 935-42.

Marcel V, Catez F, Diaz JJ (2015). p53, a translational regulator, contribution to its tumour-suppressor activity. Oncogene, 34, 5513-23

Mosmann JP, Monetti MS, Frutos MC, et al (2015). Mutation detection of E6 and LCR genes from HPV 16 associated with carcinogenesis. Asian Pac J Cancer Prev, 16, 1151-7.

Patel KR, Vajaria BN, Begum R, et al (2013). Association between $\mathrm{p} 53$ gene variants and oral cancer susceptibility in population from Gujarat, West India. Asian Pac J Cancer Prev, 14, 1093-100.

Petric M, Martinez S, Acevedo F, et al (2014). Correlation between Ki67 and histological grade in breast cancer patients treatedwith preoperative chemotherapy. Asian Pac J Cancer Prev, 15, 10277-80.

Rane CK, Minden A (2014). P21 activated kinases, structure, regulation, and functions. Small GTPases, 5, 28003.

Rivera C, Venegas B (2014). Histological and molecular aspects of oral squamous cell carcinoma (Review). Oncol Lett, 8 , $7-11$.

Sabattini E, Bisgaard K, Ascani S, et al (1998). The EnVision system, a new immunohistochemical method for diagnostics and research. Critical comparison with the APAAP,
ChemMate, CSA, LABC, and SABC techniques. J Clin Pathol, 51, 506-11.

Schliephake H (2003). Prognostic relevance of molecular markers of oral cancer-a review. Int J Oral Maxillofac Surg, 32, 233-45.

Shin MK, Kim JW (2014). Clinicopathologic and diagnostic significance of $\mathrm{p} 53$ protein expression in papillary thyroid carcinoma. Asian Pac J Cancer Prev, 15, 2341-4.

Shinohara S, Kikuchi M, Tona R, et al (2014). Prognostic impact of p16 and p53 expression in oropharyngeal squamous cell carcinomas. Jpn J Clin Oncol, 44, 232-40.

Silva Junior Jde A, Camisasca DR, Bernardo V, et al (2013). The significance of $\mathrm{p} 53$ immunoexpression with different clones (DO-7 and PAb-240) in oral squamous cell carcinoma. ORL J Otorhinolaryngol Relat Spec, 75, 82-90.

Taghavi N, Biramijamal F, Sotoudeh M, et al (2010). Association of p53/p21 expression with cigarette smoking and prognosis in esophageal squamous cell carcinoma patients. World $J$ Gastroenterol, 16, 4958-67.

Verma R, Singh A, Jaiswal R, et al (2014). Association of Ki67 antigen and p53 protein at invasive tumor front of oral squamous cell carcinoma. Indian J Pathol Microbiol, 57, 553-7.

Yan J, Liu XL, Han LZ, et al (2015). Relation between Ki-67, ER, PR, Her2/neu, p21, EGFR, and TOP II- $\alpha$ expression in invasive ductal breast cancer patients and correlations with prognosis. Asian Pac J Cancer Prev, 16, 823-9. 